\title{
Forschungsherausforderungen der Interaktion und Kooperation im Krisenmanagement
}

Im Zuge der wachsenden Vernetzung mit mobilen, interaktiven und kooperativen Technologien und der zunehmenden Bedeutung sozialer Medien haben sich auch im Bereich Krisenmanagement und zivile Sicherheitsforschung neue Möglichkeiten und Problembereiche entwickelt, die besonderer Aufmerksamkeit bedürfen. Neue Ansätze und Prototypen adressieren Interaktionen und Kooperationen innerhalb und zwischen klassischen BOS ("Behörden und Organisationen mit Sicherheitsaufgaben"), aber zunehmend auch Interaktionen und Kooperationen, welche auch die von der Krise betroffenen Akteure, wie Industrie und Bürger als aktive Krisenmanager wahrnehmen und unterstützen.

Basierend auf der Unterscheidung zwischen BOS und der Öffentlichkeit müssen dabei verschiedene Szenarien der Interaktion und Kommunikation in Krisen und Katastrophen betrachtet werden (Quarantelli, 1988; Reuter, Marx, \& Pipek, 2012). Ansätze innerhalb der BOS berücksichtigen zum einen die inter-organisationale Improvisationsarbeit von Polizei, Feuerwehr und Rettungsdienst (Ley, Pipek, Reuter, \& Wiedenhoefer, 2012), zum anderen die intra-organisationale Kollaboration zwischen Leitstellen und Einsatzkräften vor Ort mithilfe mobiler digitaler Lagekarten (Monares et al., 2011; Reuter \& Ritzkatis, 2013), mobiler Applikationen zum Anfordern von Berichten und Fotos von der Einsatzstelle (Ludwig, Reuter, \& Pipek, 2013; Singh \& Ableiter, 2009) oder Anwendungen zur Koordination im feuerwehrtechnischen Innenangriff (Ramirez et al., 2012). Um auch die Bevölkerung einzubinden, gilt es alle Betroffenen - unter Berücksichtigung möglicher Infrastrukturausfälle - geeignet zu informieren (Latonero \& Shklovski, 2011; Reuter \& Ludwig, 2013). Jenseits des passiven Informierens hat die Verbreitung interaktiver und kooperativer Medien jedoch auch bisher eher unterbetrachtete Kommunikationsbeziehungen verstärkt: Auf der öffentlichen Ebene kommunizieren Bürger und Krisenhelfer mithilfe sozialer Medien wie beispielsweise Facebook oder Twitter (Palen \& Liu, 2007) und führen reale sowie virtuelle Selbsthilfeaktivitäten durch (Heger \& Reuter, 2013). Die in sozialen Medien generierten Informationen können wiederrum ausgewertet und zur Lageeinschätzung oder Analyse in der Gefahrenabwehr nutzbar gemacht werden (Abel, Hauff, \& Stronkman, 2012).

Die in diesem Themenschwerpunkt aufgeführten Beiträge liefern Erkenntnisse für alle vier Interaktions- und Kooperationsbereiche (Reuter et al., 2012): (A) BOS-interne Kooperation, (B) Krisenkommunikation mit der Bevölkerung, (C) Selbstkoordination Betroffener und Freiwilliger sowie die (D) Integration bürgergenerierter Informationen. Während (1) Betz et al. die Kommunikation innerhalb des feuerwehrtaktischen Innenangriffs mithilfe ihres "Emergency Messenger" fokussieren, tangieren alle weiteren Beiträge mehr oder weniger die Aktivitäten der Bevölkerung in Katastrophenlagen. Am Beispiel der Anschläge in Norwegen 2011 beschreiben (2) Büscher et al. das Konzept der peripheren Kooperation. Die folgenden drei Beiträge beziehen sich auf das Hochwasser 2013 in Deutschland: (3) Kaufhold und Reuter betrachten die vernetzte Selbsthilfe und leiten Gestaltungsherausforderungen für sozialen Medien her. (4) Hofmann et al. stellen ihr App-basiertes Koordinationskonzept "Hands2Help" für freiwillige Helfer und BOS vor. (5) Zisgen et al. eruieren die Möglichkeiten zur visuellen Analytik sozialer Medien und stellen den Ansatz "Scatterblogs" dar. In den abschließenden Beiträgen betrachten (6) AlAkkad und Boden kreative Kommunikationsmöglichkeiten bei Ausfällen von Teilen der Netzinfrastruktur, und (7) Nestler die Möglichkeiten der Evaluation der Mensch-Computer-Interaktion in Krisenszenarien.

Im Einzelnen adressieren die aufgeführten Beiträge den Themenschwerpunkte in folgender Weise:

1) Matthias Betz, Tobias Dyrks und Volker Wulf (Universität Siegen) beschreiben in ihrem Beitrag "Emergency Messenger als Kommunikationskonzept für Notfallarbeit" ein komplementäres textbasiertes Funkmeldesystem, welches gestützt auf den Ergebnissen ethnographischer Studien in Kooperation mit verschiedenen deutschen Feuerwehren konzeptioniert, ge- 
staltet und evaluiert wurde, um die anstrengende, gefährliche und zeitkritische Arbeit von Atemschutztrupps im Innenangriff zu unterstützen.

2) Monika Büscher, Sung Yueh Perng und Sebastian Weise (Lancester University und National University of Ireland Maynooth) beschreiben in ihrem Beitrag "Periphere Kooperation am Beispiel der Anschläge in Norwegen 2011", wie Menschen soziale Medien während der Anschläge in Norwegen nutzten, um Hilfe zu leisten. Ausgehend von der Analyse empirischer Daten wird das Konzept der „peripheren Kooperation” mit dem Ziel erarbeitet, die öffentliche Beteiligung an der Mobilisierung von Ressourcen für die Krisenhilfe besser zu verstehen.

3) Marc-André Kaufhold und Christian Reuter (Universität Siegen) untersuchen in ihrem Beitrag "Vernetzte Selbsthilfe in Sozialen Medien am Beispiel des Hochwassers 2013" die Vernetzungs- und Hilfsaktivitäten von Bürgern. Neben der qualitativen Analyse ausgewählter, entstandener Hilfsnetzwerke (Twitter, Facebook, Google Maps) wurden zusätzlich Interviews mit Gründern von Facebook-Gruppen zum Hochwasser geführt, um zum einen aktuelle Nutzungsmuster und zum anderen Gestaltungs- und Erweiterungspotenziale für Soziale Medien im Katastrophenschutz zu identifizieren.

4) Marlen Hofmann, Hans Betke und Stefan Sackmann (Martin-Luther-Universität Halle-Wittenberg) beziehen sich in ihrem Beitrag "Hands2Help - Ein App-basiertes Konzept zur Koordination Freiwilliger Helfer" ebenfalls auf das Hochwasser 2013. Die bereits im vorherigen Beitrag dargestellte Herausforderung, die vielen spontanen Helfer zu koordinieren, wird hier zum Anlass genommen, um Anforderungen an App-basierte IT-Systeme zu diskutieren, die Einsatzleiter und Leitstellen bei der Freiwilligenkoordination unterstützen können.

5) Julia Zisgen, Julia Kern, Dennis Thom und Thomas Ertl (Bundesamt für Bevölkerungsschutz und Katastrophenhilfe und Universität Stuttgart) stellen in Ihrem Beitrag "\#Hochwasser - Visuelle Analyse von Social Media im Bevölkerungsschutz" am gleichen Beispiel vor, dass Techniken zur computergestützten explorativen Datenanalyse geeignet sind, um trotz der noch recht dünnen Datenlage in Bezug auf georeferenzierte Daten relevante Erkenntnisse zu gewinnen. Hierzu wird Scatterblogs, ein interaktives Social Media-Analysewerkzeug, vorgestellt und evaluiert.

6) Amro Al-Akkad und Alexander Boden (Fraunhofer-Institut für Angewandte Informationstechnik FIT) untersuchen in ihrem Beitrag "Kreative Nutzung der verfügbaren Netzwerkinfrastruktur im Katastrophenfall" Herausforderungen für Nutzer von mobilen Geräten in Situationen, in denen die Netzwerkinfrastruktur gestört ist. Aufbauend auf Interviews werden fünf architektonische Qualitäten, welche die Resilienz von Systemen für Krisenkommunikation bei Ausfällen der Kommunikationsinfrastruktur erhöhen können, beschrieben und anhand zweier Prototypen erläutert.

7) Simon Nestler (Hochschule Hamm-Lippstadt) rundet den Themenschwerpunkt mit seinem Beitrag "Evaluation der Mensch-Computer-Interaktion in Krisenszenarien" ab: Basierend auf eigenen Untersuchungen werden verschiedene Ansätze für das Usability-Testing von interaktiven Krisenmanagementsystemen vorgestellt. Dazu gehen diese Ansätze über eine rein methodische Betrachtung des Usability-Testings hinaus. Der Usability-Test wird aus der Perspektive der MenschComputer-Interaktion sowie aus der Perspektive des Krisenmanagements betrachtet.

Die Beiträge aus diesem Themenschwerpunkt liefern unserer Ansicht nach interessante Einblicke in die aktuelle Forschung. Wir danken allen Beitragenden, den (anonymen) Gutachtern des Peer-Reviews und dem i-com Herausgeberteam für ihren Beitrag zu diesem Themenschwerpunkt.

Volkmar Pipek und Christian Reuter

Gastherausgeber

\section{Gastherausgeber}

Volkmar Pipek ist Professor für Computerunterstützte Gruppenarbeit und Soziale Medien am Institut für Wirtschaftsinformatik der Universität Siegen. Er studierte in Kaiserslautern Informatik und Wirtschaftswissenschaften mit Schwerpunkt Daten- 
banken und Künstliche Intelligenz. Nach einer Tätigkeit als wissenschaftlicher Mitarbeiter am Institut für Informatik III der Universität Bonn arbeitete er als Gastforscher am Laboratory of $\mathrm{HCl}$ and Group Technology der Universität Oulu in Finnland und promovierte in Information Processing Science.

Christian Reuter studierte Wirtschaftsinformatik an der Universität Siegen und École Supérieure de Commerce de Dijon in Frankreich. Nach seinem Abschluss arbeitete er zuerst als IT Consultant für ein großes Telekommunikations-Unternehmen, bis er ein Promotionsstipendium erhielt und seine Tätigkeit als wissenschaftlicher Mitarbeiter im Bereich Computerunterstützte Gruppenarbeit und Soziale Medien am Institut für Wirtschaftsinformatik der Universität Siegen begann. Derzeit ist er mit den Drittmittelprojekten InfoStrom und EmerGent betraut.

\section{Literatur}

Abel, F., Hauff, C., \& Stronkman, R. (2012). Twitcident: Fighting Fire with Information from Social Web Streams. In Proceedings of the 21st international conference companion on World Wide Web (pp. 5-8). New York.

Heger, O., \& Reuter, C. (2013). IT-basierte Unterstützung virtueller und realer Selbsthilfegemeinschaften in Katastrophenlagen. In R. Alt \& B. Franczyk (Eds.), Proceedings of the International Conference on Wirtschaftsinformatik (pp. 1861-1875). Leipzig, Germany.

Latonero, M., \& Shklovski, I. (2011). Emergency Management, Twitter, and Social Media Evangelism. International Journal of Information Systems for Crisis Response and Management (IJISCRAM), 3(4), 1-16.

Ley, B., Pipek, V., Reuter, C., \& Wiedenhoefer, T. (2012). Supporting Improvisation Work in Inter-Organizational Crisis Management. In Proceedings of the Conference on Human Factors in Computing Systems (CHI) (pp. 1529-1538). Austin, USA: ACM Press.

Ludwig, T., Reuter, C., \& Pipek, V. (2013). What You See Is What I Need: Mobile Reporting Practices in Emergencies. In O. W. Bertelsen, L. Ciolfi, A. Grasso, \& G. A. Papadopoulos (Eds.), Proceedings of the European Conference on Computer Supported Cooperative Work (ECSCW) (pp. 181-206). Paphos, Cyrus: Springer.

Monares, Á., Ochoa, S. F., Pino, J. A., Herskovic, V., Rodriguez-Covili, J., \& Neyem, A. (2011). Mobile computing in urban emergency situations: Improving the support to firefighters in the field. Expert Systems with Applications, 38(2), 1255-1267. Palen, L., \& Liu, S. B. (2007). Citizen communications in crisis: anticipating a future of ICT-supported public participation. In Proceedings of the Conference on Human Factors in Computing Systems (CHI). San Jose, USA: ACM Press.

Quarantelli, E. L. (1988). Disaster Crisis Management: A summary of research findings. Journal of Management Studies, 25(4), 373-385.

Ramirez, L., Dyrks, T., Gerwinski, J., Betz, M., Scholz, M., \& Wulf, V. (2012). Landmarke: an ad hoc deployable ubicomp infrastructure to support indoor navigation of firefighters. Personal and Ubiquitous Computing (PUC), 16(8), 1025-1038.

Reuter, C., \& Ludwig, T. (2013). Anforderungen und technische Konzepte der Krisenkommunikation bei Stromausfall. In M. Hornbach (Ed.), Informatik 2013 - Informatik angepasst an Mensch, Organisation und Umwelt, GI-Edition-Lecture Notes in Informatics (LNI) (pp. 1604-1618). Koblenz, Germany: GI.

Reuter, C., Marx, A., \& Pipek, V. (2012). Crisis Management 2.0: Towards a Systematization of Social Software Use in Crisis Situations. International Journal of Information Systems for Crisis Response and Management (IJISCRAM), 4(1), 116.

Reuter, C., \& Ritzkatis, M. (2013). Unterstützung mobiler Geo-Kollaboration zur Lageeinschätzung von Feuerwehr und Polizei. In R. Alt \& B. Franczyk (Eds.), Proceedings of the International Conference on Wirtschaftsinformatik (pp. 18771891). Leipzig, Germany.

Singh, G., \& Ableiter, D. (2009). TwiddleNet: Smartphones as Personal Content Servers for First Responders. In J. Löffler \& M. Klann (Eds.), Mobile Response: Second International Workshop on Mobile Information Technology for Emergency Response (Vol. 5424, pp. 130-137). Bonn, Germany: Springer-Verlag. 